\title{
Fibrinolysis and D-dimer in COVID-19: A Twisted Plot!
}

\author{
Rohan Magoon ${ }^{1}$, Nitin Choudhary ${ }^{2}$, Jes Jose ${ }^{3} \odot$
}

\begin{abstract}
Coronavirus disease-2019 (COVID-19) and the associated pathophysiological perturbations continue to bewilder the fraternity at large. In this context, the thromboembolic predisposition in COVID-19 has particularly emerged as a matter of an ardent debate. The index commentary aims to present an account of the recent developments in the understanding of the immunothrombosis in the enigmatical setting of COVID-19. Keywords: Coronavirus disease-2019, D-dimer, Fibrinolysis, Plasminogen, Point-of-care testing, Thromboembolism.

Indian Journal of Critical Care Medicine (2022): 10.5005/jp-journals-10071-24105
\end{abstract}

\section{HighLights}

- COVID-19-associated microvascular and macrovascular thrombosis has emerged as an important contributor to the disease-related morbidity-mortality.

- While a hypercoagulable state in COVID-19 has received much attention, fibrinolytic pathway was largely presumed to be overactive given the D-dimer elevation.

- Recent demonstration of an attenuated fibrinolytic response in advanced COVID-19 disease is akin to a double-trouble situation and can have important implications.

\section{BACKGROUND}

Orchestrating a sound clinical management essentially pivots around an extensive comprehension of the underlying pathophysiology. However, the development of an ideal treatment regime against the novel coronavirus disease-2019 (COVID-19) presents unprecedented challenges, particularly when our understanding of this relentless and enigmatical disease continues to evolve.

\section{COVID-19 ImMUNOTHROMBoSIS: SIMILAR Yet Different}

While COVID-19-related thromboembolic sequel (a major contributor to morbidity-mortality) has captivated attention, ${ }^{1,2}$ the clinicopathological peculiarities surrounding a thromboinflammatory predisposition in COVID-19 are equally important and necessitate elaboration. The COVID-19 immunothrombosis emanates owing to the coagulation abnormalities resulting from the multicellular interaction of circulating leukocytes, platelets, and endothelial cells. Nevertheless, independent research groups suggest that unlike disseminated intravascular coagulation (DIC) and bacterial sepsis, the conventional coagulation tests (prothrombin time, activated partial thromboplastin time, antithrombin levels, etc.) are not significantly altered in most of the COVID-19 patients despite an elevated incidence of clinically relevant prothrombotic events. At the same time, a substantial proportion of COVID-19 patients do not fulfil the usual DIC criterion. ${ }^{3}$

\section{Viscoelastic Testing in COVID-19: Has MUCH TO SAY}

The aforementioned depiction of the lack of substantially altered standard coagulation tests highlights the subsequent importance of viscoelastic testing in delineation of the COVID-19-associated

\footnotetext{
1,2Department of Anaesthesia, Atal Bihari Vajpayee Institute of Medical Sciences and Dr Ram Manohar Lohia Hospital, New Delhi, India

${ }^{3}$ Department of Cardiac Anesthesiology, Sri Jayadeva Institute of Cardiovascular Sciences and Research, Bengaluru, Karnataka, India

Corresponding Author: Nitin Choudhary, Department of Anaesthesia, Atal Bihari Vajpayee Institute of Medical Sciences and Dr Ram Manohar Lohia Hospital, New Delhi, India, Phone: +91 8447698566, e-mail: drnitinchoudhary86@gmail.com

How to cite this article: Magoon R, Choudhary N, Jose J. Fibrinolysis and D-dimer in COVID-19: A Twisted Plot! Indian J Crit Care Med 2022;26(2):164-166.

Source of support: Nil

Conflict of interest: None
}

coagulopathy. Appropriate to the context, Bachler et al.'s and Heiz et al.'s clinical reports constitute the most recent remarkable research in this area. ${ }^{4,5}$ Bachler et al. retrospectively compared the ClotPro viscoelastic test [recombinant tissue-derived plasminogen activator ( $r$-tPA) challenge assay] findings of 20 critically ill COVID-19 patients with 60 healthy volunteers. ${ }^{4}$ They outlined a hypercoagulable state with an impaired (hypo) fibrinolysis [increased lysis time (LT) in tPA test (508 vs 210 seconds; $p<0.01$ )] in the COVID-19 cohort of patients. ${ }^{4}$ Alongside, the COVID-19 patients with an attenuated fibrinolytic response had significantly higher fibrinogen levels in comparison to those with a preserved fibrinolytic response. Interestingly, both the groups revealed a comparable profile of D-dimer elevation irrespective of the state of fibrinolysis. ${ }^{4}$

In another study by Heiz et al., aggregometric (Multiplate) and viscoelastomeric tests (CloPro) were performed in 27 COVID19 patients with acute respiratory distress syndrome (ARDS) and 12 healthy adults. ${ }^{5}$ The authors depicted a significantly greater maximum clot firmness ( $37 \pm 11$ vs $15 \pm 4 \mathrm{~mm} ; p<0.001)$ with an increased LT ( $530 \pm 327$ vs $211 \pm 80$ seconds; $p<0.001)$ in the COVID-19 group as opposed to the healthy volunteers, again strengthening the possibility of hypofibrinolysis in COVID-19 patients. $^{5}$

\section{Fibrinolysis and D-dimer Conundrum}

It is well-known that an escalated D-dimer has emerged as the most remarkable coagulation assay abnormality heralding 
thromboembolic events in COVID-19 and demonstrates a linear association with the mortality. ${ }^{3-6}$ Nevertheless, the elevated D-dimer in COVID-19 which was initially interpreted as a surrogate of an enhanced fibrinolysis in this cohort need not necessarily represents a state of hyperfibrinolysis. ${ }^{4,5,7}$ This is substantiated by the fact that the fibrinogen and thrombin concentrations have been demonstrated to be raised in COVID-19, albeit as low as $0.02 \%$ of fibrinogen is actually cleaved to D-dimers. ${ }^{7,8}$

Hence, D-dimer escalation points toward an increased fibrin deposition rather than an enhanced lysis and fails to truly reflect the fibrinolytic state (hyper, normo, or hypo). The massive fibrin production beyond the lytic capacity with the subsequent intraalveolar (leading to pulmonary intravascular coagulation, major cause of ventilation-perfusion mismatch in COVID-19 ARDS) and microcirculatory deposition accentuating the predisposition to end-organ dysfunction which is in turn is further perpetuated by the coexistent endothelial injury. ${ }^{1-3,6}$

\section{Disturbances in Tenuously Balanced Fibrinolytic Pathway in COVID-19}

The fibrinolytic pathway (plasminogen-plasmin system) plays an important role in the pathophysiology and progression of viral illnesses such as COVID-19. Albeit the plasma concentrations of tPA can be elevated up to six times in COVID-19, the lack of translation of the former into a resultant hyperfibrinolytic state (particularly in advanced stages of disease) is explained by a number of contributory factors: ${ }^{3,9}$

- First, the plasminogen-plasmin system not only cleaves fibrinogen but is also overwhelmed by additional substrates such as matrix proteins, misfolded proteins, and necrotic materials, which are quite prevalent in ARDS. ${ }^{4}$

- Second, the profibrinolytic activity in COVID-19 can be potentially undermined owing to the high levels of plasminogen activator inhibitor-1 (PAl-1) and thrombin activatable fibrinolysis inhibitor (TAFI) promoting the pendulum swing in the favor of a hypofibrinolytic state. Moreover, the increased PAI-1 detrimentally affects the fibrinolysis far ahead of the associated increase in TPA in COVID-19., 4,5-9

- Third, complement and renin-aldosterone-angiotensin system (RAAS) pathway activation in COVID-19 can also deleteriously impact the fibrinolytic mechanisms. ${ }^{9}$

- Finally, the platelets add to the insult by functioning as PAI-1 generators with ongoing inflammation, endothelial dysfunction, systemic hypoxemia, and thrombin activation (common in a COVID-19 setting) stimulating the platelets to result in enhanced PAI-1 levels in the circulation., ${ }^{1,4-9}$

\section{Evolving Clinical-therapeutic Viewpoint}

Needless to say, the above mentioned discussion has important clinical implications. It is noteworthy that hypofibrinolysis has been reported to predict sepsis-related mortality in general. ${ }^{10}$ Many physicians propose the perilous combination of $\mathrm{D}$-dimer elevation alongside fibrinolysis shutdown as the most superior predictor of COVID-19-associated thromboembolic complications. ${ }^{11}$

In an attempt to stimulate fibrinolysis in patients with fibrinolytic shutdown, administration of plasminogen and r-tPA seems to have a promising role. ${ }^{12-14}$ In a case series by Wang et al., significant improvement in oxygen saturation was seen following systemic administration of r-tPA (alteplase) which however was short-lived and imposed a significant risk of bleeding (1.2\%) as these patients are on anticoagulant therapy. ${ }^{13} \mathrm{~A}$ phase II clinical trial of r-tPA inhalation [Inhaled Tissue Plasminogen Activator for Acute Plastic Bronchitis (PLATyPuS); NCT02315898] is also underway.

In another study, Wu et al. administered atomized plasminogen in 13 COVID-19 patients and found significant improvement in oxygen saturation likely because the inhaled plasminogen dissolved the fibrin deposited along the lung parenchyma and improved alveolar ventilation. ${ }^{14}$ The plausible benefit of inhaled over systemic therapy is the decreased incidence of bleeding, but the risk associated with spread of infection following aerosol generation and the unpredictability of its systemic absorption needs to be borne in mind. Therefore, utmost care should be exercised during patient selection and meticulously administered to those in a documented hypofibrinolytic state of COVID-19. ${ }^{4,11,12}$

\section{Conclusion}

To conclude, the underlying pathophysiology leading to a thromboembolic predisposition in COVID-19 can be peculiarly complicated. A sound comprehension of the former can substantiate the prevention and management of COVID-19-related thromboembolism. Herein, a theranostic approach backed by the point-of-care coagulation testing can be pivotal in guiding a personalized management of this sick subset. ${ }^{11,15}$ This is exemplified by the aforementioned elucidation of a twisted plot of fibrinolysis and D-dimer in COVID-19.

\section{Author Contributions}

RM contributed for conceptualization and written the original manuscript. JJ contributed for data search and assisted in drafting the manuscript. NC contributed for conceptualization and assisted in drafting the manuscript.

\section{ORCID}

Rohan Magoon @ https://orcid.org/0000-0003-4633-8851

Nitin Choudhary @ https://orcid.org/0000-0002-8933-1222

Jes Jose $\odot$ https://orcid.org/0000-0003-1734-9519

\section{References}

1. Magoon R. COVID-19 related strokes: Pandora's Box may open as the p(c)lot thickens! [March 19, 2021]. Neurologia 2021;36(7):562-563. DOI: 10.1016/j.nrl.2021.03.004.

2. Magoon R. COVID-19 and congenital heart disease: cardiopulmonary interactions for the worse! Paediatr Anaesth 2020;30(10):1160-1161. DOI: 10.1111/pan.14004.

3. Levi M, Iba T. COVID-19 coagulopathy: is it disseminated intravascular coagulation? Intern Emerg Med 2021;16(2):309-312. DOI: 10.1007/ s11739-020-02601-y.

4. Bachler M, Bösch J, Stürzel DP, Hell T, Giebl A, Ströhle M, et al. Impaired fibrinolysis in critically ill COVID-19 patients. Br J Anaesth 2021;126(3):590-598. DOI: 10.1016/j.bja.2020.12.010.

5. Heinz C, Miesbach W, Herrmann E, Sonntagbauer M, Raimann FJ, Zacharowski K, et al. Greater fibrinolysis resistance but no greater platelet aggregation in critically ill COVID-19 patients. Anesthesiology 2021;134(3):457-467. DOI: 10.1097/ALN.0000000000003685.

6. Magoon R. Pulmonary vasculature in COVID-19: mechanism to monitoring! Korean J Anesthesiol 2021;74(2):186-187. DOI: 10.4097/ kja.20536. 
7. Görlinger K, Levy JH. COVID-19-associated coagulopathy. Anesthesiology 2021;134(3):366-369. DOI: 10.1097/ALN.000000 0000003688 .

8. Görlinger K, Dirkmann D, Gandhi A, Simioni P. COVID-19-associated coagulopathy and inflammatory response: what do we know already and what are the knowledge gaps? Anesth Analg 2020;131(5):1324-1333. DOI: $10.1213 /$ ANE.0000000000005147.

9. Kwaan HC, Lindholm PF. The central role of fibrinolytic response in COVID-19: a hematologist's perspective. Int J Mol Sci 2021;22(3):1283. DOI: 10.3390/ijms22031283.

10. Schmitt FCF, Manolov V, Morgenstern J, Fleming T, Heitmeier S, Uhle F, et al. Acute fibrinolysis shutdown occurs early in septic shock and is associated with increased morbidity and mortality: results of an observational pilot study. Ann Intensive Care 2019;9(1):19. DOI: 10.1186/s13613-019-0499-6.

11. Görlinger K, Almutawah H, Almutawaa F, Alwabari M, Alsultan Z, Almajed J, et al. The role of rotational thromboelastometry during the COVID-19 pandemic: a narrative review. Korean J Anesthesiol 2021;74(2):91-102. DOI: 10.4097/kja.21006.

12. Weiss E, Roux O, Moyer JD, Paugam-BurtzC, Boudaoud L, Ajzenberg N, et al. Fibrinolysis resistance: a potential mechanism underlying COVID-19 coagulopathy. Thromb Haemost 2020;120(9):1343-1345. DOI: 10.1055/s-0040-1713637.

13. Wang J, Hajizadeh N, Moore EE, McIntyre RC, Moore PK, Veress LA, et al. Tissue plasminogen activator (tPA) treatment for COVID-19 associated acute respiratory distress syndrome (ARDS): a case series. J Thromb Haemost 2020;18(7):1752-1755. DOI: 10.1111/jth. 14828.

14. Wu Y, Wang T, Guo C, Zhang D, Ge X, Huang Z, et al. Plasminogen improves lung lesions and hypoxemia in patients with COVID-19. QJM 2020113(8):539-545. DOI: 10.1093/qjmed/hcaa121.

15. Magoon R. Implications of practice variability: comment. Anesthesiology 2020;133(4):943-944. DOI: 10.1097/ALN.000000 0000003465. 